\title{
Surgical outcomes for lumbar spinal canal stenosis with coexisting cervical stenosis (tandem spinal stenosis): a retrospective analysis of 565 cases
}

Tsuyoshi Yamada, Toshitaka Yoshii ${ }^{*}$ D, Naoki Yamamoto, Takashi Hirai, Hiroyuki Inose and Atsushi Okawa

\begin{abstract}
Background: Concurrent cervical and lumbar spinal canal stenosis is known as tandem spinal stenosis (TSS). As research on TSS is limited, there is no consensus on the optimal surgical approach to this problem. We evaluated the prevalence and clinical characteristics of TSS in patients with symptomatic lumbar spinal canal stenosis (LCS).

Methods: The authors performed a retrospective analysis of the outcomes of 565 patients who underwent lumbar surgeries performed for symptomatic LCS. In all the patients, both the cervical and lumbar regions were evaluated preoperatively, and we compared TSS patients and non-TSS patients in terms of multiple clinical parameters. In the TSS patients, we investigated the ratio and clinical outcomes of additional cervical surgeries performed on TSS patients.

Results: Two hundred two cases (35.8\%) were considered to be TSS. Twenty-eight patients (5.0\%) underwent a cervical operation during the follow-up period. There were no differences between the radiographic TSS patients and non-TSS patients in terms of preoperative lumbar-Japanese Orthopedic Association (L-JOA) scores, postoperative L-JOA scores, and the L-JOA recovery rate $(14.8 \pm 4.4$ points vs $14.2 \pm 4.6$ points, $23.9 \pm 4.3$ points vs $23.1 \pm 4.5$ points, $63.7 \pm 28.2 \%$ vs $60.3 \pm 27.9 \%$, respectively), while the TSS group included a greater number of hypertension cases. The recovery rate L-JOA scores of patients who underwent additional cervical surgeries were significantly lower compared with the rate of patients who experienced treatment for only lumbar lesions ( $62.8 \pm 25.8 \%$ vs $39.8 \pm 35.5 \%, p=0.0003)$. However, additional cervical surgery still improved both the cervical myelopathy-Japanese Orthopedic Association (C-JOA) and L-JOA scores in TSS patients with symptomatic cervical lesion (from $10.3 \pm 2.8$ points to $12.1 \pm 3.0$ points, $p=0.0302$; from $14.8 \pm$ 7.3 points to $19.9 \pm 5.0$ points, $p=0.0331$, respectively). In these patients, there were no significant differences in the recovery rate of both C-JOA and L-JOA scores between the single-stage surgery group and the staged surgery group ( $40.7 \pm 35.8 \%$ vs $20.7 \pm 16.1 \% ; 50.9 \pm 25.1 \%$ vs $34.2 \pm 39.3 \%$, respectively).
\end{abstract}

Conclusions: Radiographic co-existing cervical stenosis did not affect surgical outcomes for LCS, although symptomatic cervical lesion affected neurological score after lumbar surgery. An additional surgery for cervical lesion significantly improved neurological findings in TSS patients.

Keywords: Tandem spinal stenosis, Lumbar spinal canal stenosis, Spine surgery

* Correspondence: yoshii.orth@tmd.ac.jp

Department of Orthopaedic Surgery, Graduate School, Tokyo Medical and

Dental University, 1-5-45 Yushima, Bunkyo-ku, Tokyo 113-8510, Japan International License (http://creativecommons.org/licenses/by/4.0/), which permits unrestricted use, distribution, and reproduction in any medium, provided you give appropriate credit to the original author(s) and the source, provide a link to the Creative Commons license, and indicate if changes were made. The Creative Commons Public Domain Dedication waiver (http://creativecommons.org/publicdomain/zero/1.0/) applies to the data made available in this article, unless otherwise stated. 


\section{Background}

Tandem spinal stenosis (TSS) is defined as concurrent cervical and lumbar stenosis. Dagi et al. reported that TSS was characterized by a triad of intermittent neurogenic claudication, progressive gait disturbance, and mixed myelopathy and polyradiculopathy in both the upper and lower extremities [1]. The prevalence of TSS has been reported to range from 5 to $28 \%$ [1-3]. When a TSS patient has severe cervical myelopathy, concurrent lumbar spinal canal stenosis (LCS) is often abstrused by symptoms due to a cervical lesion, leading to difficulty in identifying the origin of these neurological findings, especially in the lower limbs. Due to the complicated nature of this condition, research on TSS is limited, and thus, there is no consensus on the optimal surgical approach to this problem [4].

The surgical strategy for patients with TSS is still controversial. Predominantly, symptomatic legions are usually surgically treated. If neurological symptoms originate significantly from both the cervical and lumbar spine, then both lesions require surgical decompression. A staged procedure (cervical followed by lumbar [1,5] or vice versa [6]) or simultaneous cervical and lumbar decompression for TSS [2, 4, 5, 7] has been advocated. We previously investigated the effectiveness of additional lumbar surgery after cervical decompression for TSS patients who had predominant neurological symptoms originating from the cervical spine and found that the neurological recovery from single-stage surgery was equal to that of staged procedures [8].

In the present study, we investigated symptomatic LCS patients who required lumbar decompression surgery in order to illuminate more details on the epidemiology and pathology of TSS. We also evaluated the effectiveness of additional cervical decompression surgery after lumbar surgery for TSS patients who had predominant clinical symptoms from the lumbar spine.

\section{Methods}

The current retrospective study was approved by our institutional review board. The authors executed a retrospective analysis of the outcomes of 565 elective lumbar surgeries performed in symptomatic LCS patients from July 2010 to July 2016 who had a follow-up of more than 1 year after surgery. There were 286 females and 279 males, with an average age of $70.7 \pm 9.5$ years (32-92 years).

Clinical symptoms included neurologic claudication and/or radicular pain. In principle, we conducted myelography for all these patients before performing surgeries and evaluated not only the lumbar spine but also the cervical region. The exclusion criteria were cases with tumors of the cauda equina, vertebral compression fractures, single-level disc herniation, isolated lateral or foraminal stenosis, a history of spine surgery, and emergency cases in which myelography was not performed. Medical records of these patients were reviewed for demographics including the length of follow-up, age, gender, habit of smoking, and preoperative co-morbidities such as diabetes mellitus (DM), hypertension (HT), ischemic heart disease (IHD), hemodialysis (HD), collagen disease, obesity, and osteoporosis. The presence of a joint prosthesis (hip or knee) and computed tomography (CT)-detected cervical ossification of the longitudinal ligament (C-OPLL) were also recorded in this study.

Spinal cord and/or cauda equine compressions were evaluated using myelography and CT myelography (CTM). Before the lumbar surgeries, compressive lesions at the cervical levels were also evaluated in addition to the lumbar lesions. After myelography, CT scans were obtained using a multi-slice scanner (Aquilion64, TOSHIBA Medical System Inc., Tokyo) to obtain image data of the cervical and lumbar spine. The scanning parameters were as follows: $120 \mathrm{kV}, 100$ to $300 \mathrm{~mA}, 2-\mathrm{mm}$ thickness for slice data, and $2-\mathrm{mm}$ thickness for reconstruction. The scanning time for the cervical and lumbar spine was less than 10 s. No patients displayed neurological deterioration during the $\mathrm{CT}$ examinations.

The myelographic criterion for the diagnosis of LCS was narrowing $>50 \%$ of the dural sac. The criterion in the cervical spine was an absolute reduction of the dorsoventral dimension of the spinal canal to $10 \mathrm{~mm}$ or less and a disruption or block of contrast material flow [1]. In the CTM images, a compressive lesion of the spinal cord or cauda equina was also defined as a lesion in contact with the anterior or posterior aspect of the spinal cord or cauda equina, morphological deformity of the spinal cord, or the disappearance of the subarachnoid space [9]. In addition, ossification of the longitudinal ligament (OPLL) was defined as an ossified mass $(\geq 2 \mathrm{~mm})$ arising from the vertebrae [10].

Two independent spinal surgeons evaluated whether TSS was observed or not, and compressive lesions in the whole spine were assessed with a DICOM viewer. The evaluators reached a consensus. Before the image review, the testers read images from the same 60 patients to check the inter-observer agreement. The Kappa coefficient for the inter-observer agreement was 0.71 , which was considered to indicate substantial agreement.

The selection of the surgical method was based on multiple factors, such as the location of compressive pathology, the extent of the degenerative process, intervertebral instability, sagittal alignment of the lumbar spine, and medical conditions. In general, the patients got out of bed on postoperative day 2 and started rehabilitation therapies. For external spine stabilization, in the cases treated with cervical fusion, a Philadelphia collar was applied for 3 months. In cases receiving laminoplasty (LAMP), a soft neck collar was used until discharge. In cases treated with a 
one-level lumbar spine fusion, a soft brace was used postoperatively to immobilize the lumbar spine, but a hard brace was used in cases of multi-level fusions. In these patients, the operative procedure, operation time, blood loss in the cervical operation, and clinical outcomes were investigated. The clinical outcomes were assessed by means of the scoring system proposed by the Japanese Orthopedic Association (JOA); the recovery rate in the cervical case was determined by [(postoperative score - preoperative score $) /(17$-preoperative score $)] \times 100 \%$, and the lumbar cases were determined by [(postoperative score - preoperative score $) /(29$-preoperative score $)] \times 100 \%$ [11].

The demographics of the patients included in this study are shown in Table 1. We compared the non-TSS group with the TSS group in terms of multiple clinical parameters (Table 2). Moreover, we investigated the ratio and clinical outcomes of additional cervical surgery performed on TSS patients.

Statistical analysis was performed using an unpaired $t$ test and one-way ANOVA followed by Tukey-Kramer's post

Table 1 Demographics

\begin{tabular}{|c|c|c|}
\hline LCS case, no & & 565 \\
\hline \multirow[t]{7}{*}{ Disease type } & Hypertorophy of the flavum, no & $288(51.0 \%)$ \\
\hline & Disc herniation (> 2 levels), no & $60(10.6 \%)$ \\
\hline & Degenerative kyphosis, no & $23(4.1 \%)$ \\
\hline & Degenerative scoliosis, no & $21(3.7 \%)$ \\
\hline & Spondylolisthesis, no & $161(28.5 \%)$ \\
\hline & L-OYL, no & $11(1.9 \%)$ \\
\hline & L-OPLL, no & $1(0.2 \%)$ \\
\hline Sex, female/male, no & & $286 / 279$ \\
\hline Age, average (range) & & $70.7 \pm 9.5(32-92)$ \\
\hline YAM, average (range) & & $90.9 \pm 17.0(48-149)$ \\
\hline Surgical method & Decompression/posterior fusion, no & $222 / 343$ \\
\hline Follow-up period, average, years & & $3.2 \pm 1.6$ \\
\hline \multirow[t]{6}{*}{ Lumbar surgical outcomes } & Operative time, average (range), min & $220.5 \pm 104.0(44-749)$ \\
\hline & Blood loss, average (range), $\mathrm{mL}$ & $403.9 \pm 604.7(6-6380)$ \\
\hline & Preoperative L-JOA score, average (range), points & $14.6 \pm 4.4(-2-24)$ \\
\hline & Postoperative L-JOA score, average (range), points & $23.6 \pm 4.4(8-29)$ \\
\hline & Recovery rate of L-JOA score, average (range), \% & $62.5 \pm 28.1(-70.6-100)$ \\
\hline & Revisions, no & $60(10.6 \%)$ \\
\hline \multirow[t]{3}{*}{ TSS } & Radiological cervical stenosis, no & $202(35.8 \%)$ \\
\hline & Symptomatic cervical stenosis, no & $58(10.3 \%)$ \\
\hline & Cervical operation, no & $28(5.0 \%)$ \\
\hline \multirow[t]{11}{*}{ Preoperative comorbidities } & C-OPLL, no & $23(4.1 \%)$ \\
\hline & HT, no & $278(49.2 \%)$ \\
\hline & $\mathrm{HL}, \mathrm{no}$ & $93(16.5 \%)$ \\
\hline & DM, no & $121(21.4 \%)$ \\
\hline & Smoking, no & $159(28.1 \%)$ \\
\hline & IHD, no & $96(17.0 \%)$ \\
\hline & $\mathrm{HD}$, no & $30(5.3 \%)$ \\
\hline & Collagen disease, no & $46(8.1 \%)$ \\
\hline & Obesity, BMI > 25, no & $216(38.2 \%)$ \\
\hline & Artificial joint, TKA or THA, no & $33(5.8 \%)$ \\
\hline & Osteoporosis, YAM < 70\%, no & $68(12.0 \%)$ \\
\hline
\end{tabular}

Abbreviations: LCS lumbar spinal canal stenosis, L-OYL ossification of yellow ligament of the lumbar spine, L-OPLL ossification of the posterior longitudinal ligament of the lumbar spine, YAM young adult mean, L-JOA Japanese Orthopedic Association for lumbar spinal canal stenosis, TSS tandem spinal stenosis, C-OPLL ossification of the posterior longitudinal ligament of the cervical spine, $H T$ hypertension, $H L$ hyperlipidemia, DM diabetes mellitus, IHD ischemic heart disease, $H D$ hemodialysis, $B M I$ body mass index, TKA total knee arthroplasty, THA total hip arthroplasty 
Table 2 Comparison between cases without TSS and those with TSS

\begin{tabular}{|c|c|c|c|}
\hline & Non-TSS & TSS & $p$ \\
\hline Case, no & 363 & 202 & \\
\hline Sex, female/male, no & $190 / 173$ & $96 / 106$ & \\
\hline Decompression/posterior fusion, no & $132 / 231$ & $90 / 112$ & \\
\hline Age, average (range) & $70.0 \pm 9.8(34-88)$ & $72.0 \pm 8.8(32-92)$ & NS \\
\hline YAM, average (range) & $90.0 \pm 16.5(52-149)$ & $92.5 \pm 17.7(48-137)$ & NS \\
\hline Operative time, average (range), min & $221.4 \pm 101.7(50-746)$ & $218.8 \pm 108.3(44-749)$ & NS \\
\hline Blood loss, average (range), $\mathrm{mL}$ & $420.7 \pm 663.8(6-998)$ & $372.8 \pm 478.1(6-4150)$ & NS \\
\hline Preoperative L-JOA score, average (range), points & $14.8 \pm 4.4(0-24)$ & $14.2 \pm 4.6(-2-24)$ & NS \\
\hline Postoperative L-JOA score, average (range), points & $23.9 \pm 4.3(8-29)$ & $23.1 \pm 4.5(9-29)$ & NS \\
\hline Recovery rate of L-JOA score, average (range), \% & $63.7 \pm 28.2(-70.6-100)$ & $60.3 \pm 27.9(-57.1-100)$ & NS \\
\hline Revisions, no & $36(9.9 \%)$ & $24(11.9 \%)$ & NS \\
\hline Symptomatic cervical stenosis, no & $0(0 \%)$ & $58(28.7 \%)$ & \\
\hline Cervical operation, no & $0(0 \%)$ & $28(13.9 \%)$ & \\
\hline C-OPLL, no & $0(0 \%)$ & $23(11.3 \%)$ & \\
\hline HT, no & $166(45.7 \%)$ & $112(55.4 \%)^{*}$ & 0.0268 \\
\hline $\mathrm{HL}$, no & $59(16.2 \%)$ & $34(16.8 \%)$ & NS \\
\hline DM, no & $73(20.1 \%)$ & $48(23.8 \%)$ & NS \\
\hline Smoking, no & $96(26.4 \%)$ & $63(31.2 \%)$ & NS \\
\hline IHD, no & $61(16.8 \%)$ & $35(17.3 \%)$ & NS \\
\hline$H D$, no & $14(3.9 \%)$ & $16(7.9 \%)^{*}$ & 0.039 \\
\hline Collagen disease, no & $30(8.3 \%)$ & $16(7.9 \%)$ & NS \\
\hline Obesity, BMI > 25, no & $133(36.6 \%)$ & $83(41.0 \%)$ & NS \\
\hline Artificial joint, TKA or THA, no & $22(6.1 \%)$ & $11(5.4 \%)$ & NS \\
\hline Osteoporosis, YAM < 70\%, no & $45(12.4 \%)$ & $23(11.4 \%)$ & NS \\
\hline
\end{tabular}

Abbreviations: TSS tandem spinal stenosis, YAM young adult mean, L-JOA Japanese Orthopedic Association for lumbar spinal canal stenosis, C-OPLL ossification of the posterior longitudinal ligament of the cervical spine, $H T$ hypertension, $H L$ hyperlipidemia, DM diabetes mellitus, IHD ischemic heart disease, $H D$ hemodialysis, $B M I$ body mass index, TKA total knee arthroplasty, THA total hip arthroplasty, NS not significant

${ }^{*} p<.05$

hoc test and Fisher's exact test. All data are expressed as the mean \pm standard deviation (SD). A $p$ value less than 0.05 was considered to indicate a statistically significant difference.

\section{Results}

For the disease types, 565 LCS cases included 161 cases with spondylolisthesis, 11 cases with ossification of yellow ligament of the lumbar spine (L-OYL), and 1 case with lumbar OPLL (L-OPLL), for which we performed decompression in 222 cases and posterior fusion in 343 cases. The preoperative comorbidities included DM in 121 cases, HT in 278 cases, IHD in 96 cases, HD in 30 cases, collagen disease in 46 cases, obesity (body mass index $>25$ ) in 216 cases, and osteoporosis (young-adult mean $<70 \%)$ in 68 cases. Thirty-three patients had undergone total knee or hip arthroplasties prior to surgery for cervical lesions. There were 23 cases with CTMdetected C-OPLL (4.1\%) (Table 1).
From the radiographic findings, there were 202 cases (35.8\%) with radiological cervical spinal cord compression in LCS patients who underwent lumbar surgeries. These patients were included in the TSS group. The patients in this study tolerated the surgical procedure in the lumbar lesion well and were followed up for an average of 3.2 years (1.0-6.0 years). With regard to lumbar surgery, the average operative time was $220.5 \pm 104.0 \mathrm{~min}$. The estimated blood loss was $403.9 \pm 604.7$ g. Revised lumbar surgeries were applied in 60 of the 565 patients $(10.6 \%)$ due to perioperative complications such an infection, trouble with instrumentation, recurrence of stenosis, and adjacent segment disease (Table 1). Twenty-eight patients (5.0\%) actually underwent a cervical operation during the followup periods. Eleven patients underwent these procedures simultaneously. Seventeen patients underwent a two-stage surgery for TSS.

Second, in terms of the presence of TSS in LCS patients, there were no significant differences in sex, age, the average operative time, the average intraoperative blood loss, and 
the incidence of revision cases between the non-TSS group and the TSS group. With regard to clinical outcomes, there were no differences between the non-TSS groups and the TSS groups in terms of preoperative lumbar-Japanese Orthopedic Association (L-JOA) scores, postoperative L-JOA scores, and the L-JOA recovery rate. Substantial recovery of neurologic symptoms was observed in both groups. The TSS group contained a greater number of C-OPLL patients. The group also included a greater number of HT and HD patients. The rate of coexistent symptomatic cervical canal stenosis was up to $28.7 \%$ in the TSS group (Table 2).

Whether there was a single-stage simultaneous cervical and lumbar surgery or a staged surgery, we investigated to what extent performing a cervical operation in addition to lumbar surgery could affect the postoperative outcome of TSS patients. We divided these 202 TSS patients into two groups according to the presence of additional operations. The mean period between cervical and lumbar lesion surgeries was $1.0 \pm 1.3$ years. The mean recovery rate of cervical myelopathy-Japanese Orthopedic Association (C-JOA) scores at the final follow-up point was $28.5 \pm$ $31.3 \%$ when the operation for cervical lesions was applied simultaneously or following lumbar surgery. Postoperative L-JOA scores and the recovery rate of L-JOA scores in the patients who underwent additional cervical surgeries were significantly lower compared with the rate in the patients who experienced the treatment for only lumbar lesions $(23.5 \pm 4.3$ points vs $19.9 \pm 5.0$ points, $p=0.0005 ; 62.8 \pm$ $25.8 \%$ vs $39.8 \pm 35.5 \%, p=0.0003$, respectively). Nevertheless, in the additional cervical surgery group, the L-JOA score before cervical surgery was $14.8 \pm 7.3$ points, but it significantly improved to $19.9 \pm 5.0$ points after cervical surgery $(p=0.0331)$ (Table 3$)$. This group also showed significant improvement in C-JOA scores from $10.3 \pm 2.8$ points to $12.1 \pm 3.0$ points $(p=0.0302)$.

The present study included 30 patients who had symptomatic cervical lesions but never experienced cervical operations. Their mean recovery rate of L-JOA scores at the final follow-up was $56.4 \pm 27.0 \%$. The additional cervical operation group $(n=28)$ could not get over these outcomes $(56.4 \pm 27.0 \%$ vs $39.8 \pm 35.5 \%$, NS). Severe cervical lesions, to the extent they needed operations, could be profoundly attributed to poor outcomes after lumbar and cervical surgeries. Further, except for operative times, there were no significant differences in various clinical parameters, even in the recovery rate of both C-JOA and L-JOA scores between the single-stage surgery group and the staged surgery group (Table 4).

\section{Discussion}

Because the spondylotic process in the spine may initially present as an isolated segmental problem, there are often multiple levels of concurrent degenerative pathology [12] that are recorded as TSS. The pathology of TSS is complicated, and the interpretation of clinical outcomes seems controversial and difficult. Due to the limited number of these patients, a clearly defined prevalence and surgical algorithm do not exist. The aim of this retrospective study was to determine the prevalence of myelography/CTMdetected cervical canal stenosis that could be either asymptomatic or could manifest itself as a symptomatic cervical lesion in the patients with symptomatic LCS who underwent lumbar surgeries.

Lee et al. reported that magnetic resonance imaging (MRI)-detected moderate and severe cervical cord compression is observed in $24 \%$ of LCS patients [13]. From the radiographic findings in the present study, there were 202 TSS cases (35.8\%) in 565 LCS patients. The radiological TSS rate was higher compared with the previous report, probably because the LCS patients included in our study had relatively more severe symptoms that required surgery. The rate of coexistent clinically symptomatic cervical canal stenosis was $28.7 \%$ in the radiological TSS patients, and $13.9 \%$ of the radiological TSS cases needed actual cervical operations. These results seem to support the theory that the presence of symptomatic LCS increases the risk of spondylotic cervical cord compression [14, 15].

Most previous studies have used MRI to evaluate spinal stenosis in the cervical and lumbar spine. However, CTM offers great advantages for visualizing bony compressive lesions. In this study, we used CTM to evaluate the TSS patients because OPLL and ossification of yellow ligament (OYL) are occasionally causative factors for spinal stenosis. In both OPLL cases and spondylotic (non-OPLL) cases, the stenotic lesions, which need surgery, exist at multiple areas in the whole spine. On the other hand, the previous study reported that OPLL cases had a tendency to develop into TSS at a relatively younger age when compared with spondylotic cases [8]. There is also a difference in the distribution of stenotic lesions between the OPLL and spondylotic cases. The incidence of OPLL/OYL in the lower lumbar spine was quite low (2.1\% in this study), whereas the spondylotic process was mainly observed in the middle cervical and in the lower lumbar spine $[8,16]$.

In the evaluation of clinical symptoms, there were no differences in the L-JOA recovery rate between the nonTSS group and the TSS group. When we compared the clinical outcomes of the only lumbar operation group with those of the additional cervical operation group in TSS cases, the latter group showed a lower recovery rate of L-JOA scores, even after both lesions were completely decompressed. From these results, the radiological cervical cord compression itself (radiological TSS) does not always have an effect on clinical outcomes after lumbar surgery. However, in the TSS cases who had symptomatic cervical stenosis that needed surgery, the cervical lesion can affect the lumbar neurological score after lumbar surgeries. 
Table 3 Comparison between cases without cervical surgery and those with cervical surgery

\begin{tabular}{|c|c|c|c|}
\hline & Only lumbar operation & Additional cervical operation & $p$ \\
\hline Case, no & 174 & 28 & \\
\hline Sex, female/male, o & $83 / 91$ & $13 / 15$ & \\
\hline Decompression/posterior fusion, no & $67 / 107$ & $23 / 5$ & \\
\hline One-stage/two-stage additional operation & - & $11 / 17$ & \\
\hline Period between cervical and lumbar surgery, average, years & - & $1.0 \pm 1.3(0-5)$ & \\
\hline Age, average (range) & $72.2 \pm 8.5(32-89)$ & $70.8 \pm 10.6(43-92)$ & NS \\
\hline YAM, average (range) & $92.4 \pm 17.8(48-137)$ & $93.8 \pm 17.4(69-127)$ & NS \\
\hline Operative time, average (range), min & $221.4 \pm 108.5(44-749)$ & $201.6 \pm 107.4(70-572)$ & NS \\
\hline Blood loss, average (range), $\mathrm{mL}$ & $381.5 \pm 493.1(6-4150)$ & $315.5 \pm 366.5(70-1450)$ & NS \\
\hline Pre lumbar operative L-JOA score, average (range), points & $14.3 \pm 4.5(-2-24)$ & $13.2 \pm 5.4(-2-22)$ & NS \\
\hline Pre cervical operative L-JOA score, average (range), points & - & $14.8 \pm 7.3(3-26)$ & \\
\hline Postoperative L-JOA score, average (range), points & $23.5 \pm 4.3(9-29)^{*}$ & $19.9 \pm 5.0(11-29)$ & 0.0005 \\
\hline Recovery rate of L-JOA score, average (range), \% & $62.8 \pm 25.8(-8.3-100)^{*}$ & $39.8 \pm 35.5(-57.1-100)$ & 0.0003 \\
\hline Preoperative C-JOA score, average (range), points & - & $10.3 \pm 2.8(5-15)$ & \\
\hline Postoperative C-JOA score, average (range), points & - & $12.1 \pm 3.0(6.5-17)$ & \\
\hline Recovery rate of C-JOA score, average (range), \% & - & $28.5 \pm 31.3(0-100)$ & \\
\hline Symptomatic cervical stenosis, no & $30(17.2 \%)$ & $28(100 \%)$ & \\
\hline Revisions, no & $24(13.8 \%)$ & $0(0 \%)$ & \\
\hline C-OPLL, no & $17(9.8 \%)$ & $6(21.4 \%)$ & NS \\
\hline HT, no & 97 (55.7\%) & $15(53.6 \%)$ & NS \\
\hline$H L$, no & $26(14.9 \%)$ & $8(28.6 \%)$ & NS \\
\hline DM, no & $40(23.0 \%)$ & $8(29.3 \%)$ & NS \\
\hline Smoking, no & $57(32.8 \%)$ & $6(21.4 \%)$ & NS \\
\hline IHD, no & $31(17.8 \%)$ & $4(14.3 \%)$ & NS \\
\hline HD, no & $12(6.9 \%)$ & $4(14.3 \%)$ & NS \\
\hline Collagen disease, no & $15(8.6 \%)$ & $1(3.6 \%)$ & NS \\
\hline Obesity, BMI > 25, no & 71 (40.8\%) & $12(42.9 \%)$ & NS \\
\hline Artificial joint, TKA or THA, no & $10(5.7 \%)$ & $1(3.6 \%)$ & NS \\
\hline Osteoporosis, YAM < 70\%, no & $23(13.2 \%)$ & $0(0 \%)$ & \\
\hline
\end{tabular}

Abbreviations: YAM young adult mean, L-JOA Japanese Orthopedic Association for lumbar spinal canal stenosis, C-JOA Japanese Orthopedic Association for cervical myelopathy, C-OPLL ossification of the posterior longitudinal ligament of the cervical spine, $H T$ hypertension, $H L$ hyperlipidemia, $D M$ diabetes mellitus, $I H D$ ischemic heart disease, HD hemodialysis, BMI body mass index, TKA total knee arthroplasty, THA total hip arthroplasty, NS not significant ${ }^{*} p<.05$

Additional cervical decompression can provide some improvements in lumbar symptoms because the lumbar neural fibers may also be under compression in the cervical spondylotic processes [9]. Indeed, 28 additional cervical surgeries for TSS in our study significantly improved not only C-JOA scores but also L-JOA scores from $14.8 \pm 7.3$ points to $19.9 \pm 5.0$ points post-cervical decompression. From these findings, we concluded that the prognosis of TSS with symptomatic LCS was no worse than non-TSS, while the prognosis can be significantly influenced by symptoms of the isolated cervical stenosis. However, the additional cervical surgery can still produce some improvement in LCS patients who have symptomatic cervical lesions.
It remains unknown which procedure, two-staged or single-stage surgery, is more effective for achieving postoperative neurologic improvement in TSS patients. When both cervical and lumbar stenoses appeared equally symptomatic, we generally address the cervical spine first or choose a simultaneous procedure. However, in patients included in this study, most cases have severer symptoms in the lumbar spine compared to the cervical spine. Therefore, we performed lumbar surgery first for the patients included in this study. In some limited patients (1.9\%), we chose a simultaneous procedure. However, neurological recovery of simultaneously single-stage surgery in TSS was equal to that of staged surgery in the present study. Furthermore, as described above, 28 
Table 4 Comparison between cases with single-stage operations and those with two-stage operations

\begin{tabular}{llll}
\hline & Single-stage & Two-stage \\
\hline Case, no & 11 & 17 & $9 / 8$ \\
Sex, female/male, no & $4 / 7$ & $12 / 5$ & $1.7 \pm 1.3(0.2-5)$ \\
Decompression/posterior fusion, no & $11 / 0$ & $73.4 \pm 10.4(44-85)$ \\
Period between cervical and lumbar surgery, average, years & 0 & $62.2 \pm 16.9(69-123)$ \\
Age, average (range) & $97.4 \pm 19.6(69-127)$ & $168.6 \pm 74.2(70-349)$ \\
YAM, average (range) & $257.6 \pm 134.1(117-572) *$ & $253.8 \pm 282.4(10-1208)$ \\
Operative time, average (range), min & $432.3 \pm 486.6(70-1450)$ & $12.8 \pm 5.9(-2-22)$ \\
Blood loss, average (range), mL & $14.0 \pm 4.5(8-21)$ & $14.8 \pm 7.3(3-26)$ \\
Pre lumbar operative L-JOA score, average (range), points & - & $19.0 \pm 5.2(11-28)$ \\
Pre cervical operative L-JOA score, average (range), points & $21.7 \pm 4.3(18-29)$ & $34.2 \pm 39.3(-57.1-90.9)$ \\
Postoperative L-JOA score, average (range), points & $50.9 \pm 25.1(21.4-100)$ & $10.2 \pm 2.9(5-15)$ \\
Recovery rate of L-JOA score, average (range), \% & $10.5 \pm 2.6(7-13)$ & $11.7 \pm 3.0(6.5-16)$ & NS \\
Preoperative C-JOA score, average (range), points & $12.7 \pm 3.1(7-15.5)$ & $20.7 \pm 16.1(0-80)$ & NS \\
Postoperative C-JOA score, average (range), points & $40.7 \pm 35.8(0-100)$ & NS \\
Recovery rate of C-JOA score, average (range), \% & NS & NS & NS \\
\hline
\end{tabular}

Abbreviations: YAM young adult mean, L-JOA Japanese Orthopedic Association for lumbar spinal canal stenosis, C-JOA Japanese Orthopedic Association for cervical myelopathy, NS not significant

${ }^{*} p<.05$

patients in our study received additional cervical surgery after averaged 1.0 year later than the first lumbar surgery and showed significant neurological recovery not only in cervical neurological scores but also in lumbar scores. These results suggested that cervical operations can be staged if the single-stage surgery is considered to be too invasive for the patient. Eskander et al. reported that age increased the risk of major and minor complications regardless of the surgical algorithm chosen to manage TSS [4]. Our previous study revealed that TSS with myelopathy was associated with age, HT, and DM, whereas TSS with symptomatic LCS was related to HT and HD in the present study. TSS could be related to lifestyle-related disease. Therefore, surgical management should be tailored to the patient's age and general condition, whether it is by simultaneous or staged procedures.

This study has several other limitations, including the following: (1) the patient baseline was not controlled; (2) this was a retrospective investigation; and (3) this was not population-based. These limitations could be partially ascribed to the challenges of TSS-related surgery. Prospective data collection is needed in the future to clarify more precise clinical outcomes in TSS cases. We must also take the potential risk of radiation by $\mathrm{CT}$ scanning into consideration. All patients in this study underwent CTM as a preoperative examination to determine the approach or the vertebral level at which the surgical procedure should be performed.

The subjects in the previous study were limited to TSS cases with myelopathy in which the operations of cervical lesions were prior to lumbar lesions [8]. The present study approached the cases in which lumbar decompressions are prior to cervical lesions to disclose TSS epidemiology and pathology beyond this limitation. The study indicated that the severity of cervical lesions could have a more critical impact on neurological improvement after lumbar surgery for TSS. Given these results combined with our previous data, the authors concluded that TSS could be associated with lifestyle-related disease and that an additional surgery for another lesion significantly improved neurological findings in TSS patients.

\section{Conclusion}

Radiographic co-existing cervical stenosis did not affect surgical outcomes for LCS, although symptomatic cervical lesion affected neurological score after lumbar surgery. An additional surgery for cervical lesion improved neurological findings in TSS.

\section{Abbreviations \\ CT: Computed tomography; CTM: CT myelography; DM: Diabetes mellitus; HD: Hemodialysis; HT: Hypertension; IHD: Ischemic heart disease; JOA: The Japanese Orthopedic Association; LAMP: Laminoplasty; LCS: Lumbar spinal canal stenosis; MRI: Magnetic resonance imaging; NS: Not significant; OPLL: Ossification of the longitudinal ligament; OYL: Ossification of yellow ligament; TSS: Tandem spinal stenosis}

\section{Acknowledgements}

Not applicable.

\section{Funding}

This work was supported by the Japanese Health Labour Sciences Research Grant (038). 


\section{Availability of data and materials}

The datasets used and/or analyzed during the current study are available from the corresponding author on reasonable request.

\section{Authors' contributions}

TY1 participated in the data collection, data analysis, and manuscript preparation. TY2 participated in the study design, data collection, data analysis, and manuscript preparation. NY participated in the data collection. TH participated in the data collection and data analysis. $\mathrm{HI}$ participated in the data collection. AO participated in the study design and manuscript preparation. All authors read and approved the final manuscript.

\section{Ethics approval and consent to participate}

The current retrospective study was approved by the institutional review board (Tokyo Medical and Dental University). The informed consent was obtained from each patient.

\section{Consent for publication}

Not applicable.

\section{Competing interests}

Atsushi Okawa is currently receiving a science research grant (038) from the Japanese Health Labour. For the remaining authors, none were declared.

\section{Publisher's Note}

Springer Nature remains neutral with regard to jurisdictional claims in published maps and institutional affiliations.

Received: 8 February 2018 Accepted: 12 March 2018

Published online: 20 March 2018

\section{References}

1. Dagi TF, Tarkington MA, Leech JJ. Tandem lumbar and cervical spinal stenosis. Natural history, prognostic indices, and results after surgical decompression. J Neurosurg. 1987;66:842-9.

2. Kikuike K, Miyamoto K, Hosoe H, Shimizu K. One-staged combined cervical and lumbar decompression for patients with tandem spinal stenosis on cervical and lumbar spine: analyses of clinical outcomes with minimum 3 years follow-up. J Spinal Disord Tech. 2009;22:593-601.

3. LaBan MM, Green ML. Concurrent (tandem) cervical and lumbar spinal stenosis: a 10-yr review of 54 hospitalized patients. Am J Phys Med Rehabil. 2004;83:187-90.

4. Eskander MS, Aubin ME, Drew JM, et al. Is there a difference between simultaneous or staged decompressions for combined cervical and lumbar stenosis? J Spinal Disord Tech. 2011;24:409-13.

5. Naderi S, Mertol T. Simultaneous cervical and lumbar surgery for combined symptomatic cervical and lumbar spinal stenoses. J Spinal Disord Tech. 2002;15:229-31.

6. Aydogan M, Ozturk C, Mirzanli C, Karatoprak O, Tezer M, Hamzaoglu A. Treatment approach in tandem (concurrent) cervical and lumbar spinal stenosis. Acta Orthop Belg. 2007;73:234-7.

7. Krishnan A, Dave BR, Kambar AK, Ram H. Coexisting lumbar and cervical stenosis (tandem spinal stenosis): an infrequent presentation. Retrospective analysis of single-stage surgery (53 cases). Eur Spine J. 2014;23:64-73.

8. Yamada T, Yoshii T, Yamamoto $\mathrm{N}$, et al. Clinical outcomes of cervical spinal surgery for cervical myelopathic patients with coexisting lumbar spinal canal stenosis (tandem spinal stenosis): a retrospective analysis of 297 cases. Spine (Phila Pa 1976). 2018:43:E234-41.

9. Miyazaki M, Kodera R, Yoshiiwa T, Kawano M, Kaku N, Tsumura H. Prevalence and distribution of thoracic and lumbar compressive lesions in cervical spondylotic myelopathy. Asian Spine J. 2015;9:218-24.

10. Kawaguchi Y, Nakano M, Yasuda T, et al. Characteristics of ossification of the spinal ligament; incidence of ossification of the ligamentum flavum in patients with cervical ossification of the posterior longitudinal ligament -analysis of the whole spine using multidetector CT. J Orthop Sci. 2016;21:439-45.

11. Hirabayashi K, Miyakawa J, Satomi K, Maruyama T, Wakano K. Operative results and postoperative progression of ossification among patients with ossification of cervical posterior longitudinal ligament. Spine (Phila Pa 1976). $1981 ; 6: 354-64$
12. Matsumoto M, Okada E, Toyama Y, Fujiwara H, Momoshima S, Takahata T. Tandem age-related lumbar and cervical intervertebral disc changes in asymptomatic subjects. Eur Spine J. 2013;22:708-13.

13. Lee SH, Kim KT, Suk KS, et al. Asymptomatic cervical cord compression in lumbar spinal stenosis patients: a whole spine magnetic resonance imaging study. Spine (Phila Pa 1976). 2010;35:2057-63.

14. Adamova B, Bednarik J, Andrasinova T, et al. Does lumbar spinal stenosis increase the risk of spondylotic cervical spinal cord compression? Eur Spine J. 2015:24:2946-53.

15. van Eck CF, Spina lii NT, Lee JY. A novel MRI classification system for congenital functional lumbar spinal stenosis predicts the risk for tandem cervical spinal stenosis. Eur Spine J. 2017;26:368-73.

16. Hirai T, Yoshii T, Iwanami A, et al. Prevalence and distribution of ossified lesions in the whole spine of patients with cervical ossification of the posterior longitudinal ligament a multicenter study (JOSL CT study). PLoS One. 2016;11:e0160117.

\section{Submit your next manuscript to BioMed Central and we will help you at every step:}

- We accept pre-submission inquiries

- Our selector tool helps you to find the most relevant journal

- We provide round the clock customer support

- Convenient online submission

- Thorough peer review

- Inclusion in PubMed and all major indexing services

- Maximum visibility for your research

Submit your manuscript at www.biomedcentral.com/submit
Biomed Central 